\title{
Rehabilitación de praderas degradadas en el trópico de México
}

Javier Francisco Enríquez Quiroz ${ }^{\mathrm{a}^{*}}$

Valentín Alberto Esqueda Esquivel ${ }^{b}$

Daniel Martínez Méndez ${ }^{\mathrm{c}}$

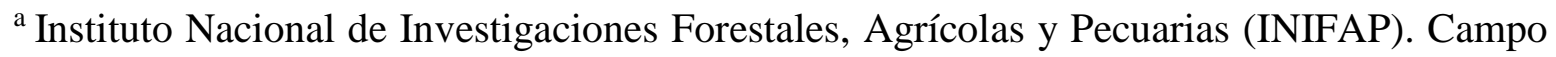
Experimental La Posta, Km. 22.5 Carretera Federal Veracruz-Córdoba. 94277, Medellín, Veracruz, México.

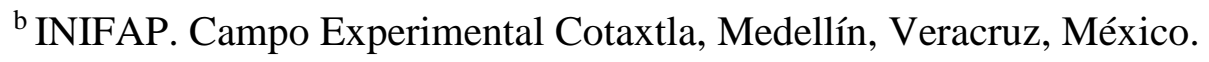

${ }^{\mathrm{c}}$ Centro de Bachillerato Tecnológico Agropecuario 251. General Felipe Ángeles, Oaxaca, México.

*Autor de correspondencia: enriquez.javier@inifap.gob.mx

\section{Resumen:}

En México, se destinan alrededor de 108.9 millones de hectáreas a la actividad ganadera, que representan el $55.5 \%$ del territorio nacional. La región del trópico cubre aproximadamente una cuarta parte de la superficie del país y en ella, la ganadería bajo pastoreo es una de las actividades económicas de mayor importancia. Se estima que, en al menos 24 estados del país, el número de cabezas de ganado, es superior a la capacidad de carga, en función de la producción de forrajes. Esta situación trae como consecuencia la degradación paulatina de las praderas y, por consiguiente, una disminución de su productividad. Una consecuencia de lo anterior es una reducción de los productos y servicios que se obtienen de ellas, como forraje, agua y áreas de recreación, asociado a una disminución en la producción de carne y leche. La investigación para la rehabilitación de praderas degradadas en el país es una actividad que se ha venido realizando en los últimos 10 años, enfocada principalmente a la eliminación de maleza por medios mecánicos y químicos, los cuales han dado resultados satisfactorios a corto plazo. Sin embargo, el problema de la degradación en México, al igual 
que en otros países tropicales, continúa por fallas en el manejo adecuado de las praderas, causados por una sobrecarga animal superior a la capacidad productiva que pueden mantener los forrajes bajo pastoreo. Esta revisión, presenta un panorama mundial y nacional de la degradación de praderas, enfocado principalmente a regiones tropicales, así como la presentación de los resultados de investigación desarrollados por el INIFAP para la recuperación de praderas, haciendo un análisis de las perspectivas en este tema a mediano y largo plazo.

Palabras clave: Ganadería, Pastos, Fertilidad del suelo, Sobrepastoreo, Control de maleza.

Recibido: 23/11/2020

Aceptado: 25/02/2021

\section{Introducción}

Debido al constante aumento de la población mundial, existe la necesidad de incrementar la producción de alimentos, pues para el año 2050 seremos 9.6 millardos de habitantes (contra 7 millardos de hoy), y este incremento se debe alcanzar con menos recursos disponibles y contaminando menos ${ }^{(1)}$. En los últimos 40 años, la producción mundial de carne se ha incrementado $90 \%$; específicamente, en el trópico, el incremento ha sido de hasta $200 \%{ }^{(1)}$. En México, se destinan alrededor de 108.9 millones de hectáreas a la actividad ganadera, que representan el $55.5 \%$ del territorio nacional ${ }^{(2)}$. Se tiene una población bovina de 32.6 millones de cabezas, lo que resalta la importancia de esta actividad económica ${ }^{(3)}$. La ganadería se desarrolla en todos los ecosistemas del país, y tiene alta relevancia en las zonas tropicales secas y húmedas, en donde se produce una cantidad importante de carne y leche que representan el 40 y $18 \%$, respectivamente, de la producción nacional ${ }^{(4)}$, en una superficie del alrededor de 56 millones de hectáreas, de las cuales se utilizan más de 23 millones de hectáreas para pastoreo ${ }^{(5)}$. En estas regiones, la fuente principal de alimento del hato bovino son los forrajes que se producen en las praderas, y que los animales consumen directamente, siendo el medio más barato para transformar a los pastos en alimentos de alta calidad nutritiva, como la carne y la leche. Se estima que, en al menos 23 entidades, el número de cabezas de ganado, es superior a la capacidad de carga que presentan las praderas en función de la producción de forrajes ${ }^{(6)}$. Esta situación ocasiona la degradación paulatina de las praderas y, por consiguiente, una disminución de su productividad, cuya consecuencia es una reducción de los productos y servicios que se obtienen de ellas, como forraje, agua y áreas de recreación, asociada a una disminución en la producción de carne y leche. El objetivo de esta revisión, es describir los factores que inciden en la degradación de praderas y las 
alternativas de solución con que se cuenta, además de mostrar los resultados obtenidos por el INIFAP en la rehabilitación de praderas en el trópico mexicano.

\section{Panorama mundial de las praderas}

Las praderas son un tipo de vegetación donde predominan los pastos o gramíneas; éstas se encuentran presentes en los cinco continentes, cubren una cuarta parte de la superficie terrestre, y contribuyen a los medios de vida de más de 800 millones de personas ${ }^{(7)}$. La fuente principal de alimentación para los sistemas de producción animal con rumiantes, son los forrajes que producen las praderas nativas, cultivadas y tierras dedicadas a la agricultura ${ }^{(8)}$. Por esta razón, su uso será fundamental para alimentar a los nueve mil millones de personas que habitarán el planeta Tierra en $2050^{(9)}$.

Las praderas proporcionan cubierta al suelo para evitar erosión eólica e hídrica, ofrecen lugares de recreación y son hábitat de especies que se utilizan para fines ornamentales y medicinales ${ }^{(10)}$. También los pastos ayudan en la captación de agua ${ }^{(11)}$, sobre todo cuando se conservan en buena condición, ya que mejoran su infiltración ${ }^{(12)}$. Asimismo, las praderas tienen potencial para la captura de carbono, sobre todo cuando se tiene un pastoreo moderado $^{(12)}$, aspecto que se incrementa si se asocian con leguminosas ${ }^{(13)}$.

\section{Panorama nacional}

En México, la superficie dedicada a la actividad ganadera, se conforma de 1.4 millones de ranchos, corrales de engorda, empresas integrales y otras unidades económicas involucradas principalmente en la producción de ganado bovino ${ }^{(3)}$. La producción de carne en canal para 2019 fue de 2,027 mil toneladas, con un consumo per cápita de 14.9 kilogramos. A su vez, la producción de leche fue de 12,275 millones de litros y ocupa el lugar $16^{\circ}$ de la producción mundial, con un consumo per cápita de $95.1 \mathrm{~L}$.

En el ámbito nacional, la producción de forrajes y pecuaria tienen una participación importante por subsector en la actividad productiva a del país, de 42 y $8 \%$, respectivamente; del total, la región noroeste produce el 7 y $6 \%$, el noreste el 24 y $22 \%$, el centro occidente 37 y $43 \%$, el centro 11 y $12 \%$ y el sureste 20 y $16 \%$, respectivamente ${ }^{(2)}$. En las regiones tropicales, existen cuatro estados que dedican más del $50 \%$ de su superficie a las actividades ganaderas: Tabasco con $65.7 \%$, Tamaulipas con $58.2 \%$, Sinaloa con $50.6 \%$ y Veracruz con $50.2 \%$. 


\section{Producción nacional de forrajes}

La producción anual de forrajes en el país, es de 183 millones de toneladas de materia seca. En términos generales, el $42 \%$ de éstos se produce en praderas, el $29 \%$ en pastizales nativos, el $24 \%$ se obtiene de esquilmos agrícolas y el $4.9 \%$ por cultivos forrajeros ${ }^{(14)}$. Se debe considerar que, tanto los pastos nativos, como las praderas perennes aportan el $71 \%$ del forraje total (136 millones de toneladas); sin embargo, sólo se debe utilizar un máximo de $60 \%$ (82 millones de toneladas) de este recurso, considerando un manejo adecuado. Por otra parte, tanto los cultivos forrajeros y los esquilmos agrícolas pueden utilizarse al $100 \%$ (55 millones de toneladas). Por lo tanto, el forraje utilizable en México es de 137 millones de toneladas. Se estima que, 34 millones de unidades animales existentes en el país, utilizan alrededor de 170 millones de toneladas de materia seca al año. Consecuentemente, existe una deficiencia de forraje de 33 millones de toneladas. Por lo anterior, el exceso de unidades animales está causando una sobreexplotación de las tierras de pastoreo, con el consecuente daño y deterioro de los recursos naturales ${ }^{(15)}$.

\section{Regiones tropicales}

Las áreas tropicales húmedas de México ocupan una superficie de 23.9 millones de hectáreas ${ }^{(16)}$. Se caracterizan por tener precipitaciones de más de 1,300 milímetros anuales y menos de 1,000 metros de altitud; en estas condiciones se desarrolla la ganadería de carne y doble propósito, en praderas con una proporción alta de pastos introducidos o $\operatorname{mejorados}^{(16,17)}$. Por otra parte, la superficie de las áreas que conforman el trópico seco es de 31.7 millones de hectáreas. En éstas, se tienen precipitaciones de 600 a 1,300 milímetros anuales y altitudes desde el nivel del mar hasta los 2,000 metros; su uso principal es el desarrollo de ganadería bovina productora de becerros para engorda ${ }^{(16,17)}$. En ambas regiones, los inventarios de las superficies con praderas introducidas y particularmente con pastos del género Brachiaria, han ido creciendo desde 1999, año en que inició la comercialización de semilla botánica. De acuerdo a los volúmenes de semilla comercializados, en 2004 se estimaba una superficie de 2`616,130 ha establecidas con estos pastos en el trópico mexicano $^{(18,19)}$. De 2004 a 2020, la superficie de nuevas praderas ha cambiado sustancialmente, ya que año con año se siembran diferentes especies y cultivares, como Meghatyrsus maximus (Jacq.) B. K. Simon \& S. W. L. Jacobs, que también tiene un mercado importante con sus cultivares Mombasa y Tanzania. Así también, otros pastos se establecen con material vegetativo, como los forrajes de corte de la especie Cenchrus purpureus (Schumach.) Morrone, que tiene varios cultivares que se siembran intensivamente, al igual que los pastos pangola (Digitaria eriantha Steud.) y estrella de África [Cynodon plectostachyus (K. Schum.) Pilg.]. Todas estas especies y cultivares por su mayor capacidad productiva y calidad con relación a las gramas nativas, han ido mejorando la productividad y producción ganadera de las zonas tropicales. 


\section{Problemática general}

Se prevé que el consumo mundial de carne y productos lácteos aumente en un $173 \%$ y un $158 \%$ de 2010 a 2050, y un aumento aún mayor en el consumo de estos alimentos se espera para los países en desarrollo ${ }^{(20)}$. Esto requerirá aumentos en la disponibilidad de alimentos para animales, que, a su vez, conduciría a la conversión de biomas de alto valor, en tierras de pastoreo, lo que ejercerá una fuerte presión que podría causar sobrepastoreo en el sistema de producción ganadera basado en pasturas nativas o cultivadas ${ }^{(21)}$. De hecho, en las últimas décadas, las praderas se han degradado debido al pastoreo excesivo, el cual representa la causa principal de daño entre todos los biomas importantes. Algunos autores estimaron que alrededor del $20 \%$ de los pastos globales y el $73 \%$ de los pastos nativos están degradados ${ }^{(22)}$. En Centroamérica, se estima que entre 50 y $80 \%$ de las áreas con pasturas se encuentran en un estado avanzado de degradación, con una carga animal inferior al $40 \%$ con relación a las praderas recién establecidas, y que reciben un manejo apropiado ${ }^{(23)}$. Así mismo, se estima que la tasa de degradación de las praderas es de $12 \%$, mientras que la tasa de renovación es del $5 \%$, situación que indica que más praderas siguen degradándose en relación a las existentes ${ }^{(23,24)}$. Considerando que la sobreutilización de los pastos nativos y praderas representa uno de los problemas que limita la productividad en los sistemas vaca-cría y de doble propósito, la rehabilitación de praderas degradadas es una de las líneas prioritarias de investigación del Programa de Forrajes y Pastizales del INIFAP ${ }^{(25)}$.

\section{Causas de la degradación de praderas}

El proceso de degradación de praderas por mal manejo, inicia con una pérdida de vigor de las plantas, las cuales presentan hojas angostas, bajo índice de verdor y disminución en su capacidad de rebrote (Figura 1). Como consecuencia, se presenta pérdida de la cobertura aérea de la especie forrajera, dejando espacio para el desarrollo de malezas, o suelo descubierto, que propicia la compactación por el pisoteo de los animales y la erosión ${ }^{(26,27)}$.

Figura 1: Causas de la degradación de las praderas tropicales

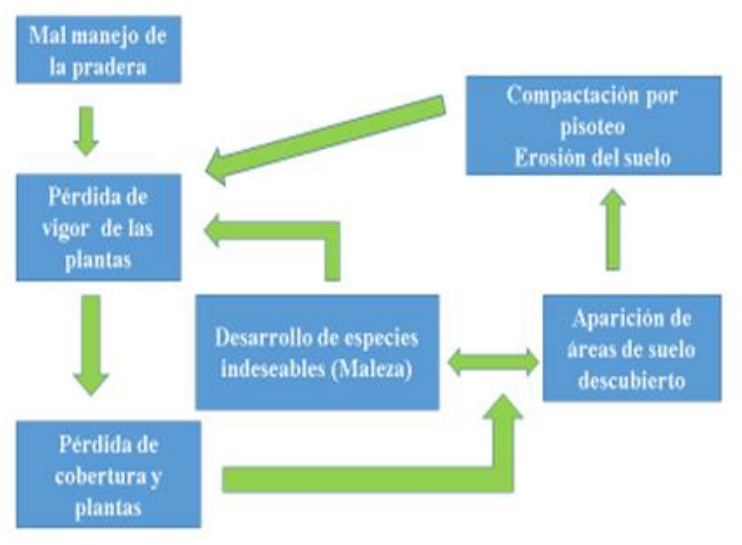


Los criterios para calificar un pastizal como degradado, son: a) disminución de la producción y calidad del forraje, b) disminución de la cobertura vegetal y densidad de plantas, c) bajo número de plantas nuevas provenientes de la resiembra natural, d) procesos de erosión del suelo por la acción de las lluvias, e) presencia de malezas de hoja ancha y angosta que no son consumidas por los animales, y f) colonización por gramíneas nativas ${ }^{(27)}$. El grado de degradación de un pastizal se puede caracterizar por el porcentaje del área ocupada por las plantas invasoras; los niveles de degradación se clasifican en: a) praderas productivas, con 0 a $10 \%$ del área con especies invasoras, b) degradación leve, con 11 a $35 \%$, c) degradación moderada, con 36 a $60 \%$ y d) degradación avanzada, con 61 a $100 \%{ }^{(27)}$. Otro criterio de clasificación se basa en establecer cuatro niveles en una clasificación cualitativa que incluye el color de la planta; los criterios cuantitativos involucran los porcentajes de material muerto, de suelo desnudo y de malezas, así como la edad de pradera (Cuadro 1).

Cuadro 1: Descripción cualitativa y cuantitativa de niveles de degradación de praderas

\begin{tabular}{|c|c|c|c|c|}
\hline \multirow[b]{2}{*}{ Síntoma } & \multicolumn{4}{|c|}{ Nivel de degradación } \\
\hline & $\begin{array}{c}1 \\
\text { No aparente }\end{array}$ & $\begin{array}{c}2 \\
\text { Bajo }\end{array}$ & $\begin{array}{c}3 \\
\text { Moderado }\end{array}$ & $\begin{array}{c}4 \\
\text { Severo }\end{array}$ \\
\hline Color de planta & $\begin{array}{l}\text { Verde } \\
\text { oscuro }\end{array}$ & Verde claro & Verde amarillo & Amarillo \\
\hline Materia muerta, \% & $<10$ & $11-20$ & $21-30$ & $>30$ \\
\hline Suelo descubierto, $\%$ & $<10$ & $11-20$ & $21-30$ & $>30$ \\
\hline Malas hierbas, $\%$ & $<10$ & $\begin{array}{c}11-20 \\
\text { Malezas de } \\
\text { hoja estrecha }\end{array}$ & $\begin{array}{c}21-30 \\
\text { Malezas de hoja } \\
\text { ancha }\end{array}$ & $\begin{array}{c}>30 \\
\text { Con pastos } \\
\text { nativos }\end{array}$ \\
\hline $\begin{array}{l}\text { Edad, años } \\
\text { establecidos }\end{array}$ & $1-3$ & $4-6$ & $7-9$ & $>10$ \\
\hline
\end{tabular}

De esta forma, el nivel 1= "No aparente", involucra praderas de uno a tres años de establecidas, color verde intenso de las hojas y valores menores al $10 \%$ de los criterios indicados, y un nivel 4= "Severo", involucra a praderas con más de 10 años de establecimiento, color amarillo de las hojas y valores superiores al $30 \%$ en el resto de los criterios, además de una alta colonización de gramíneas nativas ${ }^{(23)}$.

La productividad de una pradera puede disminuir por efecto de varios factores causantes de la degradación, como: uso de especies no aptas para las condiciones ambientales, mal manejo del pastoreo (caracterizado por el sobrepastoreo, en especial en los periodos de baja precipitación), incidencia de plagas y enfermedades, establecimiento en zonas con suelos frágiles, agotamiento de los nutrientes del suelo ocasionado por la extracción de nutrientes (el cual es mayor con especies mejoradas) y bajo o nulo uso de fertilizantes, elevada infestación de malezas herbáceas y arbustivas, y quemas indiscriminadas ${ }^{(28,29,30)}$. El pobre 
manejo de las praderas, especialmente la baja utilización de fertilizantes y el sobrepastoreo, eventualmente resultan en un decremento en la tasa de crecimiento de las gramíneas, principalmente debido a una deficiencia de nitrógeno y fósforo en el suelo ${ }^{(31)}$. La degradación de las praderas trae como consecuencia, reducción en la producción animal e incremento de costos; es decir, un problema económico y también, ecológico ${ }^{(27)}$.

\section{Vida útil de las praderas}

En la región amazónica de Brasil, después del establecimiento, la producción de una pradera disminuye gradualmente bajo condiciones de manejo tradicional; de esta forma, la vida de una pradera tiene cuatro fases, las cuales son: 1) fase de productividad alta, con cargas $>1.5$ unidades animal (UA), 2) fase de productividad media, con cargas $>1$ UA, 3) fase de productividad baja, con cargas $=0.5$ UA y 4 ) fase de degradación, con carga animal $<0.3$ UA. La primera fase tiene una duración de entre 3 y 5 años después del establecimiento de la pradera, la segunda etapa de 4 a 7 años, la tercera de 7 a 10 años, y la cuarta, de 7 a 15 años $^{(32)}$. En Honduras, se estima que las praderas tienen una vida útil de 10 años, con diferencias entre especies, ya que para Brachiaria humidicola (Rendle) Schweick. y Digitaria swazilandensis Stent es de nueve años y para C. plestostachyus es de 12 años $^{(23)}$. En México no hay información publicada al respecto; sin embargo, por observaciones personales y pláticas con productores, la vida útil de una pradera de $D$. eriantha varía de 8 a 10 años, mientras que para $B$. humidicola, $B$. decumbens y $B$. brizantha es de más de 10 años, posiblemente por las diferencias que existen en los requerimientos de fertilidad de los suelos y su manejo. Es decir, $D$. eriantha es una especie con requerimientos de fertilidad alta, $B$. brizantha requiere suelos de fertilidad media y $B$. humidicola se desarrolla en suelos de baja fertilidad; por tanto, para su mejor desempeño, cada especie debe establecerse en suelos adecuados a sus necesidades de fertilidad del suelo para su mejor desempeño productivo. En general, la vida útil de las praderas tropicales sin un apropiado manejo, podría ser en promedio de ocho años.

\section{Estrategias para la rehabilitación de praderas tropicales}

Para la recuperación de praderas se deben tener en cuenta los factores físico-químicos del suelo, la especie vegetal, y el grado de degradación de las especies que se desean restaurar. El tratamiento de recuperación de una pradera degradada y su costo depende de su grado de degradación. Cuando el proceso de degradación no es muy avanzado ( $<10 \%$ de malezas de hoja ancha), se pueden aplicar prácticas para recuperar la capacidad de producción de la pradera, pero cuando es severo, la opción más viable es el establecimiento de una nueva pradera. Algunas de las prácticas que se utilizan para incrementar la población y producción de la especie deseable, son labores agrícolas para mejorar las propiedades físicas del suelo, fertilización, control de malezas y resiembra ${ }^{(27)}$. 
La fertilización es un factor importante para la rehabilitación de praderas, ya que después de utilizar los pastos bajo pastoreo o corte por muchos años, los suelos se empobrecen, la producción de biomasa empieza a declinar y los pastos son reemplazados por otras especies. En una pradera que tenía $20 \%$ de cobertura de $B$. decumbens y el resto, ocupado por pastos nativos ${ }^{(33)}$, se evaluaron diversos tratamientos, que incluyeron sistemas de preparación o remoción del suelo, más fertilización con $22 \mathrm{~kg} \mathrm{ha}^{-1}$ de fósforo solamente y fórmulas completas $\left(22,45,25,28\right.$ y $15 \mathrm{~kg} \mathrm{ha}^{-1}$ de $\mathrm{P}, \mathrm{N}, \mathrm{K}, \mathrm{CaO}, \mathrm{MgO}$ y S, respectivamente). Se encontró que la fertilización tuvo un efecto favorable en la cobertura promedio de $B$. decumbens con $72 \%$, mientras que en el testigo sin fertilizantes se redujo a $18 \%$. Las producciones de forraje fueron de $844,3,386$ y $4,266 \mathrm{~kg} \mathrm{ha}^{-1}$ para el testigo sin fertilización, fertilización con sólo fósforo y fertilización completa, respectivamente. Cabe señalar que los sistemas de preparación y remoción del suelo no mejoraron la recuperación de la pradera, posiblemente porque estos funcionan mejor en plantas de crecimiento estolonífero, ya que la remoción propicia la resiembra de estolones en el terreno.

En otro estudio con una pastura degradada de $B$. decumbens, después de 10 años de uso, se evaluó la aplicación de macro y micronutrientes y rastreo del terreno. Se encontró un mejor efecto en la recuperación cuando se aplicaron macro y micronutrientes, mientras que el paso de rastra, afectó negativamente el desarrollo de raíces y la producción de materia seca, y no tuvo efecto en la recuperación de la pastura ${ }^{(34)}$. Los autores indicaron que la respuesta negativa se debió a la destrucción de plantas por el proceso de labranza, lo que provocó que no hubiera respuesta a la aplicación de fertilizantes.

La labranza por sí sola no tuvo efectos significativos sobre la recuperación de praderas en suelos deficientes de nutrientes, por lo que esta práctica se debe complementar con la aplicación de fertilizantes después del laboreo del suelo. Se ha demostrado que la aplicación de tratamientos mecánicos sin fertilización, no mejora el desarrollo de la pastura ni su productividad $^{(35)}$. Con D. eriantha y $C$. plectostachyus, especies que se reproducen por estolones, un paso de rastra puede ser un método adecuado para recuperar praderas sembradas, ya que su uso ocasiona una resiembra en el terreno.

El uso de leguminosas se ha indicado como una práctica viable para la rehabilitación de praderas de B. brizantha degradadas. En Brasil, la siembra manual de leguminosas y fertilización con $50 \mathrm{~kg}$ de fósforo, incrementaron la producción de materia seca ${ }^{(36)}$. En otro estudio, en una pastura degradada de Hyparrhenia rufa (Nees) Stapf, con más de 15 años de establecida y con 60 a $70 \%$ de cobertura de malezas, se probaron diferentes métodos de recuperación. Estos métodos incluyeron:1) control de maleza, 2) control de maleza + fertilización con fósforo + leguminosas, 3) control de maleza + siembra de B. humidicola + leguminosas, manejo del pastoreo con carga baja y alta, con pastoreo continuo y rotacional. Los resultados indicaron que el método más eficiente para recuperar o sustituir pasturas degradadas de $H$. rufa fue el control de maleza + siembra de $B$. humidicola + leguminosas, 
con el cual se produjo mayor cantidad y composición química del forraje, se tuvo mayor carga animal y mejores ganancias de peso $^{(37)}$. De esta forma, B. humidicola por su agresividad y amplia adaptación, así como una mejor calidad del forraje debido a su asociación con leguminosas, proporcionó resultados superiores en la producción animal, con relación al resto de los tratamientos.

\section{Rehabilitación de potreros mediante el control químico de malezas}

Una de las características de las praderas degradadas es la presencia de altas poblaciones de malezas, que ocupan los espacios que van dejando los pastos, por lo que, eventualmente la competencia por agua, luz y nutrientes se vuelve crítica ${ }^{(38)}$. Las malezas pueden aprovechar estos recursos mejor que los pastos, ya que agrupan a diversas especies, con diferentes necesidades y habilidades y una distribución espacial y estado de desarrollo no uniformes ${ }^{(39)}$, por lo que tienen la capacidad de explorar más eficientemente el ambiente en busca de los factores esenciales para el crecimiento, reduciendo su disponibilidad para los $\operatorname{pastos}^{(40,41)}$.

Aunque en una pradera se pueden presentar malezas monocotiledóneas y dicotiledóneas, generalmente, estas últimas son más importantes, debido a que tienen mayor diversidad y frecuencia de aparición $^{(42,43)}$, aunque en ocasiones las malezas gramíneas pueden llegar a ser dominantes $^{(44)}$.

La competencia de las malezas ocasiona reducción en el desarrollo y vigor de los pastos, lo cual se refleja en menor rendimiento de forraje. En ensayos realizados en tres localidades con climas Aw del centro y norte del estado de Veracruz, con los pastos Digitaria decumbens Stent., Andropogon gayanus Kunth. y C. plectostachyus se determinó que, en diferentes etapas de evaluación, la competencia ininterrumpida de las malezas, ocasionó reducciones en la producción de biomasa seca de los pastos, de 54 a $80 \%$ en $D$. decumbens ${ }^{(45)}$, de 61 a $81 \%$ en ${\text { A. } \text { gayanus }^{(46)} \text { y de } 57 \text { a } 84 \% \text { en C. plectostachyus }}^{(47)}$. Como consecuencia de dicha competencia, en $A$. gayanus se observó una reducción significativa en el contenido de proteína cruda en el muestreo realizado a los 163 días después de la aplicación (dda). Por su parte, en $C$. plectostachyus, se detectaron reducciones de contenido de proteína cruda a los 155 y 224 dda.

En una siembra del pasto Urochloa brizantha (Hochst. ex A. Rich) R. D. Webster, establecida en Mato Grosso, Brasil ${ }^{(48)}$, se reportó una reducción de $30.8 \%$ de la altura de pasto y de $9.5 \%$ en el número de macollos cuando se permitió la competencia de las malezas por 15 días a partir de la emergencia (dpe); cuando el periodo de competencia se amplió a 60 dpe, la reducción en ambos parámetros fue de 51.1 y $35.7 \%$, respectivamente. Lo anterior se reflejó en reducciones en la biomasa seca total del pasto de 50.2 y $69 \%$ con periodos de competencia de 15 y 60 dpe, respectivamente. También en Brasil, con el mismo pasto ${ }^{(49)}$ se 
determinó que la competencia de la maleza redujo el valor del radio hoja/tallo, siendo la reducción directamente proporcional al periodo con competencia; además, el contenido de proteína cruda del pasto se redujo entre 7 y $33 \%$ con periodos de competencia de 60 o más dpe.

La reducción de la productividad de los pastos debido a la competencia de las malezas es el factor que más impacto económico ocasiona, ya que disminuye el forraje disponible para alimentar al ganado. Sin embargo, algunas especies de malezas también pueden ocasionar heridas a los animales por la presencia de espinas o tricomas urticantes o intoxicación por consumo $^{(50,51)}$.

En vista de los fuertes problemas agronómicos, económicos y de salud del ganado que ocasionan las malezas, es necesario controlarlas oportunamente, antes de que afecten la productividad y calidad del pasto. El periodo en que éstas se deben controlar varía de acuerdo a las especies y densidad de población de las malezas presentes, de la variedad de pasto y de las condiciones agroclimáticas, especialmente temperatura y humedad. En condiciones de clima cálido, para $U$. brizantha, se indica que las medidas de control de malezas se deben realizar a más tardar a los $9^{(52)}, 15^{(48)}$ o $30^{(49)}$ días de coexistencia pasto-malezas, mientras que para U. ruziziensis (R. Germ. \& C. M. Evrard) Crins, se recomienda controlar la maleza antes de los 22 días de coexistencia ${ }^{(53)}$.

Los métodos que más se utilizan para el control de malezas en pastizales y potreros son los chapeos manuales o mecánicos y la aplicación de herbicidas selectivos. Los chapeos no eliminan por completo a las malezas, afectan tanto a éstas como a los pastos y sólo tienen un efecto temporal. Por su parte, los herbicidas proporcionan un control más eficiente que los chapeos, porque pueden eliminar completamente a las malezas sin ocasionar daños significativos a los pastos. En los potreros y pastizales se utilizan principalmente los herbicidas 2,4-D, picloram, fluroxipir, aminopiralid y triclopir, que se aplican en postemergencia solos o en mezcla y actúan como reguladores del crecimiento, y metsulfurón metil, que es un inhibidor de la síntesis de aminoácidos ${ }^{(54)}$. Es importante aclarar que, para evitar o minimizar afectaciones al medio ambiente, los herbicidas se deben utilizar de manera racional, aplicándolos en las dosis y épocas recomendadas en sus etiquetas. Además, los aplicadores deben utilizar las prendas de protección apropiadas para reducir los riesgos de contaminación o intoxicación.

En México se han documentado varios casos sobre uso de herbicidas en la rehabilitación de praderas degradadas en el trópico, los cuales se mencionan a continuación:

En el municipio de Medellín, Ver., en una pradera con cobertura inicial de $U$. brizantha de $27 \%, 15 \%$ de otros pastos, $56 \%$ de malezas y de suelo desnudo de $2 \%$, se realizó control químico de malezas. Los tratamientos incluyeron la aplicación de los herbicidas 2,4-D, 
picloram $+2,4-\mathrm{D}$, metsulfurón metil y aminopiralid + metsulfurón metil, los cuales en promedio redujeron la cobertura de malezas a $3.8 \%$ desde los 30 días después de la aplicación, e incrementaron la de $U$. brizantha a $88 \%$, la cual llegó a hasta $98.3 \%$ a los 75 dda. A su vez, la cobertura de malezas del tratamiento de chapeo, fue de $67 \%$ a los 30 dda y de $33 \%$ a los 75 dda, siendo las coberturas de $U$. brizantha de 12 y $54 \%$ a los 30 y 75 dda, respectivamente. Lo anterior se reflejó en producciones promedio de biomasa seca de $U$. brizantha a los $75 \mathrm{dda}$, entre 5,475 y $6,381 \mathrm{~kg} \mathrm{ha}^{-1}$ para los tratamientos de control químico y de $1,448 \mathrm{~kg} \mathrm{ha}^{-1}$ para el chapeo ${ }^{(55)}$.

En el municipio de Medellín, Ver., algunos autores ${ }^{(56)}$ indicaron que en una parcela de pasto pangola (D. eriantha) con una cobertura inicial de $23 \%$ y de acahual (Baltimora recta L.) de $33 \%$, la aplicación de metsulfurón metil, 2,4-D y la mezcla formulada de picloram + 2,4-D ofrecieron controles del acahual superiores a $90 \%$ a partir de los 30 dda. Con ello se llegó a un rendimiento promedio de forraje seco de $D$. eriantha de $51.9 \%$ superior al que se obtuvo en el testigo sin aplicación.

En trabajos que se establecieron en una pradera degradada de C. plectostachyus ${ }^{(57)}$ se evaluó el efecto de la aplicación de las mezclas formuladas de picloram + 2,4-D, aminopiralid + 2,4D y aminopiralid + fluroxipir-meptil + 2,4-D en el control de escobilla (Sida acuta Burm. f.), malva de cochino (Sida rhombifolia L.) y piñon negro (Jatropha gossypifolia L.), cuya cobertura inicial era de $66.3,62.5$ y $42.5 \%$, respectivamente. Se encontró que, para las tres especies de malezas, los controles más altos se obtuvieron con la mezcla de aminopiralid + fluroxipir-meptil + 2,4-D, cuya producción promedio de materia seca de pasto a los 45 dda fue 22.8 y $15.2 \%$ mayor que las que se obtuvieron con picloram + 2,4-D y aminopiralid + 2,4-D, respectivamente y $199 \%$ mayor, que la del testigo sin aplicación. Con el análisis de la información sobre el control de malezas, se puede afirmar que el control químico de malezas es la estrategia más práctica e importante para la rehabilitación de praderas tropicales, ya que los niveles de control son muy superiores a los obtenidos con métodos manuales o mecánicos, lo que se refleja en mayor producción y calidad del forraje.

\section{Retos y perspectivas en México en rehabilitación de praderas}

A corto plazo (5 años). Se espera que la información sobre la rehabilitación de praderas que se ha generado, se difunda en las diferentes regiones del trópico, para atenuar los impactos de la degradación de praderas; esto debe de estar en estrecha relación con los ajustes de carga animal y manejo del pastoreo, que es la causa principal de la degradación de praderas, por lo que se requiere de capacitación a técnicos y productores en estos dos temas. También es importante el realizar estudios sobre el impacto económico y social que la degradación de praderas tiene en la actividad ganadera del país y definir las pérdidas económicas y el costo social que se derivan de esta condición. 
A mediano plazo (10 años). Es un hecho que en el trópico mexicano la expansión y apertura para nuevas praderas en forma masiva, se iniciaron con el Programa Nacional de Desmontes realizado por la Secretaría de Agricultura y Ganadería, en los años setentas; es decir, en algunos casos, ya son 50 años de uso. Por esta razón, se requiere realizar estudios sobre otros factores que inciden en la degradación de praderas, tales como, la pérdida de fertilidad y empobrecimiento del suelo que se genera después de utilizar las praderas bajo corte o pastoreo, sin la retribución de nutrimentos. Esto se considera como una de las causas más importantes en la degradación de praderas. Otro aspecto estrechamente relacionado con la degradación de praderas es la compactación que causan los animales, pues disminuye la penetración de las raíces en el suelo, así como las tasas de infiltración de agua y causa erosión laminar en los terrenos. Por ello, es necesario realizar estudios de métodos de rehabilitación con el uso de implementos agrícolas que descompacten el suelo, los cuales se acompañen de la corrección de deficiencias nutricionales, ya sea con productos químicos, orgánicos o biológicos (leguminosas), a fin de mantener las praderas en un entorno estable y sostenible de la productividad.

A largo plazo (20 años). Se pretende tener autosuficiencia para atender la demanda nacional de carne y leche para la población mexicana, así como ofrecer productos inocuos con valor agregado, para incrementar las utilidades económicas de esta actividad, sin causar deterioro de los recursos naturales. Las nuevas tecnologías se convertirán en herramientas para revertir los impactos que ha causado la degradación de las praderas por el manejo deficiente en forma continua. Para lograr lo anterior, se requiere de un equipo de investigación multidisciplinario y recursos económicos suficientes a largo plazo, así como la infraestructura necesaria para desarrollar investigación tecnológica innovadora, aplicable a las condiciones del trópico mexicano.

\section{Conclusiones}

La degradación de praderas tropicales en México es consecuencia de una continua sobreexplotación, al alimentar con o en ellas un mayor número de animales que los que éstas pueden mantener y no reintegrar los nutrientes (a través de la fertilización), que se extraen del suelo al consumir los pastos. El control químico de las malezas ha demostrado ser el método más eficiente para la rehabilitación de praderas degradadas, pudiendo tener nuevamente praderas con alta producción de forraje en uno o dos ciclos de aplicar herbicidas selectivos. Sin embargo, después de la rehabilitación, es necesario establecer un pastoreo acorde a la época de producción de forraje y carga animal soportada y recuperar los nutrientes perdidos debido al pastoreo, mediante la aplicación de fertilizantes químicos u orgánicos. Todavía existen muchos factores relacionados con la productividad de las praderas que no se han estudiado a fondo, como la fertilización de mantenimiento de las praderas de acuerdo con los requerimientos nutricionales de la especie o también se requiere investigar en 
sistemas silvopastoriles que representan una oportunidad para tener sistemas sostenibles de producción animal con pasturas en el trópico, así como investigación en ranchos ganaderos, y capacitación a técnicos y productores. Por lo anterior, es necesario abrir esquemas de investigación integral en manejo y rehabilitación de praderas, con el fin de desarrollar una ganadería económica y ecológicamente rentable.

\section{Agradecimientos}

Hacemos patente nuestro agradecimiento a ex investigadores del INIFAP que contribuyeron con algunos estudios sobre la rehabilitación de praderas degradadas en la Península de Yucatán: Dr. Fernando Rivas Pantoja † y M.C. Javier Enrique Castillo Huchim.

\section{Conflictos de interés}

Los autores declaran que no tienen conflictos de interés con respecto al trabajo presentado en este reporte.

\section{Literatura citada:}

1. Quero CAR, Enríquez QJF, Bolaños AED, Villanueva AJF. Forrajes y pastoreo en México tropical. In: González PE, Dávalos FJL, coords. Estado del arte sobre investigación e innovación tecnológica en ganadería bovina tropical. 2a. ed. México, DF: REDGATRO, UNAM, INIFAP, CONACYT. 2018:66-91.

2. GCMA. Grupo Consultor de Mercados Agrícolas. Índex Agropecuario de México. https://gcma.com.mx/descargas/index-agropecuario/. Consultado 25 Oct, 2020.

3. SIAP. Servicio de Información Agropecuaria y Pesquera. Población y producción ganadera https://www.gob.mx/siap/documentos/poblacion-ganadera-136762. Consultado 8 Oct, 2020.

4. Román PE, Rodríguez ChMA, Aguilera SR, Ribera VGH. La transferencia de tecnología bovina en las regiones tropicales de México. In: González PE, Dávalos FJL, coords. Estado del arte sobre investigación e innovación tecnológica en ganadería bovina tropical. 2a. ed. México, DF: REDGATRO, UNAM, INIFAP, CONACYT. 2018:331-343.

5. González PE. Presentación y resumen del documento del Estado del Arte de la Red de Investigación e Innovación Tecnológica para la Ganadería Bovina Tropical (REDGATRO). In: González PE, Dávalos FJL, coords. Estado del Arte de la Red de Investigación e Innovación Tecnológica para la Ganadería Bovina Tropical. 2a. ed. México, DF: REDGATRO, UNAM, INIFAP, CONACYT. 2018:20-43. 
6. SEMARNAT. Informe de la situación del medio ambiente en México. Compendio de estadísticas ambientales. Indicadores clave, de desempeño ambiental y de crecimiento verde. Edición 2015. SEMARNAT. México. 2016.

7. Blair J, Nippert J, Briggs, J. Grassland ecology. In: Monson RK, editor. Ecology and the environment. The Plant Sciences Vol. 8. New York, NY, USA: Springler; 2014:399423.

8. Silveira PL da, Maire V, Schellberg J, Louault F. Grass strategies and grassland community responses to environmental drivers: a review. Agron Sustain Dev 2015;35:1297-1318.

9. Smith J, Tarawali S, Grace D, Sones K. Feeding the world in 2050: trade-offs, synergies and tough choices for the livestock sector. In: Michalk DL, et al, editors. Proc 22nd international grassland congress. New South Wales, Australia. 2013:1-9.

10. Nábrádi A. The economic value of grassland products. APSTRACT 2007;(1-2):19-28.

11. Havstad, KM, Peters DPC, Skaggs R, Brown J, Bestelmeyer, B, Frederickson E, et al. Ecological services to and from rangelands of the United States. Ecol Ecom 2007;64:261-268.

12. Flores AE, Frías HJ, Jurado GP, Figueroa CJD, Olalde PV, Valdivia FAG. Influencia del gatuño en la infiltración de agua y cantidad de forraje en pastizales con diferente grado de disturbio en el altiplano central mexicano. Tec Pecu Mex 2006;44(1):27-40.

13. Fisher MJ, Rao IM, Ayarza MA, Lascano CE, Sanz JI, Thomas RJ, et al. Carbon storage by introduced deep-rooted grasses in the South American savannas. Nature 1994;371:236-238.

14. Bolanos-Aguilar E, Emile JC, Enríquez-Quiroz JF. Les fourrages au Mexique: ressources, valorisation et perspectives de recherche. French J Graslland Forages. Fourrages 2010(204):277-282.

15. Villegas DG, Bolaños MA, Olguín PL. La ganadería en México. Colección Temas Selectos de Geografía de México. México, DF. Universidad Nacional Autónoma de México; 2001.

16. Jaramillo VV. Revegetación y reforestación de las áreas ganaderas en las zonas tropicales de México. México, DF: Secretaría de Agricultura y Recursos Hidráulicos. 1994.

17. Villegas DG. Agostaderos de México. Retrospectiva, estado actual y perspectivas [tesis maestría]. Colegio de Postgraduados; 1999. 
18. Holmann F, Rivas L, Argel P, Pérez E. Impacto de la adopción de pastos Brachiaria en Centroamérica y México. Documento de Trabajo No. 197. Cali, Colombia: CIAT. DICTA. ILRI; 2004a.

19. Holmann F, Argel P, Lascano CE. Adoption of Brachiaria grasses in Mexico and Central America: A successful story. In: McGilloway DA editor. Grassland a global resource. XX Int Grasslands Cong. Wageningen, The Netherlands. 2005:343-346.

20. Kwon Ho-Y, Nkonya E, Johnson T, Graw V, Kato E, Kihiu E. Global estimates of the impacts of grassland degradation on livestock productivity from 2001 to 2011. In: Nkonya E, et al, editors. Economics of land degradation and improvement - A global assessment for sustainable development. Heidelberg, NY, USA: Springer Open. 2016:197-214.

21. Asner G, Archer R. Livestock and carbon cycle. In: Steinfeld H, et al, editors. Livestock in a changing landscape. Vol. 1. Drivers, consequences and responses. Washington, DC, USA: Island Press; 2010;69-82.

22. Steinfeld H, Gerber P, Wassenaar T, Castel V, Rosales M, de Haan C. Livestock's long shadow: environmental issues and options. Rome, Italy, FAO; 2006.

23. Holmann F, Argel P, Rivas L, White D, Estrada RD, Burgos C et al. ¿Vale la pena recuperar pasturas degradadas? Una evaluación desde la perspectiva de productores y extensionistas en Honduras. Documento de Trabajo No. 196. Cali, Colombia: CIAT. DICTA. ILRI; $2004 b$.

24. Betancourt H, Pezo DA, Cruz J, Beer J. Impacto bioeconómico de la degradación de pasturas en fincas de doble propósito en El Chal, Petén, Guatemala. Pastos y Forrajes 2007;30(1):169-175.

25. INIFAP. Instituto Nacional de Investigaciones Forestales, Agrícolas y Pecuarias. Programa de desarrollo del INIFAP, 2018-2030. Mayor productividad en armonía con el medio ambiente. México, DF: SAGARPA. INIFAP. 2018.

26. Rincón CA. Degradación y recuperación de praderas en los llanos orientales de Colombia. Boletín Técnico No. 19. Villavicencio, Meta, Colombia: CORPOICA; 1999.

27. Padilla C, Crespo G, Sardiñas Y. Degradación y recuperación de pastizales. Rev Cubana Cienc Agríc 2009;43(4):351-354.

28. Spain JM, Gualdrón R. Degradación y rehabilitación de pasturas. In: Lascano CE, Spain $\mathrm{J}$, editores. Establecimiento y renovación de pasturas: conceptos, experiencias y enfoques de investigación. VI reunión del comité asesor de la Red Internacional de Evaluación de Pastos Tropicales. Cali, Colombia. 1991:269-283. 
29. Modesto JMS, Mascarenhas RE. Levantamento da infestação de plantas daninhas associada a uma pastagem cultivada de baixa produtividade no nordeste paraense. Planta Daninha 2001;19(1):11-21.

30. Silva MC, Ferreira SMV, Batista DJC, Lira MA, Ydoyaga SDF, Farias I, et al. Avaliação de métodos para recuperação de pastagens de braquiária no agreste de Pernambuco. 1. Aspectos quantitativos. R Bras Zootec 2004;33(6):1999-2006. Supl.

31. Boddey RM, Macedo R, Tarré RM, Ferreira E, de Oliveira OC, Rezende C de P, et al. Nitrogen cycling in Brachiaria pastures: the key to understanding the process of pasture decline. Agric Ecosyst Environ 2004;103(2):389-403.

32. Serrão SEA. Pastagem em área de floresta no trópico úmido Brasileiro: conhecimentos atuais. In: $1^{\circ}$ simposio do trópico úmido. Belem, Pa, Brasil: EMBRAPA-CPATU. 1986:147-174.

33. Arruda GN de, Cantarutti RB, Moreira EM. Tratamentos fisico-mecânicos e fertilização na recuperação de pastagens de Brachiaria decumbens em solos de tabuleiro. Pasturas Trop 1987;9(3):36-39.

34. Soares FCV, Monteriro AF, Corsi M. Recuperação de pastagens degradadas de Brachiaria decumbens. 1. Efeito de diferentes tratamentos de fertilização e manejo. Pasturas Trop 1992;14(2):2-6.

35. Carvalho SID, Vilela L, Spain JM, Karia CT. Recuperação de pastagens degradadas de Brachiaria decumbens cv. Basilisk na região dos Cerrados. Pasturas Trop 1990;12(2):24-28.

36. Costa NL, Towsend CR, Maagalhaes JA. Métodos de introdução de leguminosas em pastagens degradadas de Brachiaria brizantha cv. Marandu. Pasturas Trop 2003;25(3):39-41.

37. Gonçalves CA, Cruz OJR da, Dutra S. Recuperação e manejo de pastagens de capim jaraguá (Hyparrhenia rufa) em Rondônia Brasil. Pasturas Trop 2002;24(2):47-56.

38. Esqueda EVA, Tosquy VOH, Rosales RE. Efectividad de la mezcla picloram y fluroxipir en el control de malezas perennes de pastizales tropicales. Agron Mesoam 2005;16(2):187-192.

39. Kruchelski S, Szymczak LS, Deiss L, Moraes A. Panicum maximum cv. Aries establishment under weed interference with levels of light interception and nitrogen fertilization. Planta Daninha 2019;37:e019188589. 
40. Souza Filho APS, Veloso CAC, Gama JRN. Capacidade de absorção de nutrientes do capim-marandu (Brachiaria brizantha) e da planta daninha malva (Urena lobata) em função do pH. Planta Daninha 2000;18(3):443-450.

41. Ruas RAA, Lima JCL, Appelt MF, Dezordi LR. Controle de Brachiaria decumbens Stapf com adição de ureia à calda do glifosato. Pesq Agropec Trop 2012;42(4):455-461.

42. Guevara S, Meave J, Moreno-Casasola P, Laborde J, Castillo S. Vegetación y flora de potreros en la sierra de Los Tuxtlas, México. Acta Bot Mex 1994;28:1-27.

43. Lara JFR, Macedo JF, Brandão M. Plantas daninhas em pastagens de várzeas no estado de Minas. Planta Daninha 2003;21(1):11-20.

44. Galvão AKL, Silva JF, Albertino SMF, Monteiro GFP, Cavalcante DP. Levantamento fitossociológico em pastagens de várzea no estado do Amazonas. Planta Daninha 2011;29(1):69-75.

45. Esqueda EVA, Tosquy VOH. Efectividad de métodos de control de malezas en la producción de forraje del pasto Pangola (Digitaria decumbens Stent.). Agron Mesoam 2007;18(1):1-10.

46. Esqueda EVA, Montero LM, Juárez LFI. El control de arvenses en la productividad y calidad del pasto Llanero. Agron Mesoam 2010;21(1):145-157.

47. Esqueda EVA, Montero LM, Juárez LFI. Efecto de métodos de control de malezas en la productividad y calidad del pasto Estrella de África (Cynodon plectostachyus (K. Schum.) Pilg.). Trop Subtrop Agroecosys 2009;10(3):393-404.

48. Marchi SR, Bellé JR, Foz CH, Ferri J, Martins D. Weeds alter the establishment of Brachiaria brizantha cv. Marandu. Trop Grass1 - Forrajes Trop 2017;5(2):85-93.

49. Bellé JR, Marchi SR, Martins D, Souza AC, Pinheiro GHR. Nutritional value of Marandú palisade grass according to increasing coexistence periods with weeds. Planta Daninha 2018;36:e018170348.

50. Tuffi SLD, Santos IC, Oliveira CH, Santos MV, Ferreira FA, Queiroz DS. Levantamento fitossociológico em pastagens degradadas sob condições de várzea. Planta Daninha 2004;22(3):343-349.

51. Carvalho RM, Pimentel, RM, Fonseca DM, Santos MER. Caracterização de perfilhos em relação à planta daninha no pasto de capim-braquiária. Bol Ind Animal 2016;73(2):103-110. 
52. Jakelaitis A, Gil JO, Simões LP, Souza KV, Ludtke J. Efeitos da interferência de plantas daninhas na implantação de pastagem de Brachiaria brizantha. Rev Caatinga 2010;23(1):8-14

53. Lourenço AA, Mota RV, Sanches JL, Marques RF, Marchi SR. Weed interference in the establishment of Urochloa ruziziensis. Planta Daninha 2019;37:e019184957.

54. Enríquez QJF, Esqueda EVA, Rivas PFA, Castillo HJE, Martínez MD, López GI, et al. Rehabilitación y mejoramiento de tierras de pastoreo en el trópico de México. Folleto Técnico Núm. 79. Medellín de Bravo, Ver.: INIFAP. CIRGOC. Campo Experimental La Posta; 2015.

55. Martínez-Méndez D, Enríquez-Quiroz JF, Ortega-Jiménez E, Esqueda-Esquivel VA, Hernández-Garay A, Escalante-Estrada JAS. Rehabilitación de una pradera de pasto Insurgente con diferentes métodos de manejo. REMEXCA 2016;7(8):1787-1800.

56. Enríquez QJF, Martínez MD, Esqueda EVA, Hernández GA. Control químico de maleza para rehabilitación de una pradera de pasto Pangola. In: Toca RJA, et al. compiladores: VI congreso internacional de manejo de pastizales. Durango, Dgo.2015:109-113.

57. Esqueda EVA, Enríquez QJF. Efecto de herbicidas en el control de malezas y la producción de forraje en praderas tropicales. In: Delgado CJC, et al. editores. XXXIX Congreso mexicano de la ciencia de la maleza. Aguascalientes, Ags. 2018:137-141. 